\author{
Wilhelm G. Jacobs \\ Fakultät für Philosophie \\ Ludwig-Maximilians-Universität in München \\ jacobs.wg@gmx.de
}

\title{
SCHELLINGS REFLEXION DES KANTISCHEN BEGRIFFS WEISHEIT
}

\section{Zusammenfassung}

Kant bestimmt Weisheit als Erkenntnis des und Angemessenheit des Willens zum höchsten Gut. Dieses versteht er als Vereinigung von Moralität und Glück. Da diese nicht aus derselben Gesetzlichkeit zu begreifen sind, denkt Kant zur Vermittlung die Postulate Unsterblichkeit und Gott. Die Idee des höchsten Guts praktisch, d. i. für die Maxime unseres vernünftigen Verhaltens, hinreichend zu bestimmen, ist für Kant die Weisheitslehre, wissenschaftlich reflektiert die Philosophie. Schelling schließt sich dem an; auch für ihn ist Philosophie Wollen der Weisheit. Diese sieht auf das letzte Ziel, auf das, was in Wahrheit sein soll. Dieses zu wissen und zum bestimmenden Grund des Willens zu machen ist für Schelling Weisheit.

\section{Schlüsselwörter:}

Friedrich Wilhelm Joseph Schelling, Immanuel Kant, die Weisheit

Schelling bezieht sich in seinen Schriften immer wieder auf die Werke Kants, so auch wenn er von Weisheit spricht. Diesem Bezug soll hier nachgegangen werden.

Wort und Begriff Weisheit findet man bei Kant selten, man könnte fast meinen, wie nebenbei. Diese Meinung aber täuscht; von Weisheit spricht Kant zwar in der Tat selten, aber dafür an um so gewichtigerer Stelle. Dies möchte ich in dem ersten Teil meines Beitrags in Erinnerung rufen, um dann im zweiten von Schellings Reflexion zu sprechen. 


\section{KANTS REDE VON WEISHEIT}

In seiner Kritik der praktischen Vernunft bedeutet Weisheit für Kant „theoretisch betrachtet, die Erkenntniß des höchsten Guts und praktisch die Angemessenheit des Willens zum höchsten Gute. " ${ }^{\text {11 }}$ Weisheit bezieht sich gemäß dieser Stelle nicht auf die Erkenntnis und die Erfüllung der Pflicht, sondern auf das höchste Gut. Die Forderung der praktischen Vernunft ist unbedingt gewiß, ob sie befolgt wird oder nicht - darüber läßt Kant keinen Zweifel. Erfüllt aber das vernünftige Wesen diese Forderung, und zwar um ihrer selbst willen, so wird es würdig, glücklich zu sein. Daher hat mit der sittlichen Willensbestimmung die praktische Vernunft noch nicht ihr Ziel vollständig erreicht; dieses ist das der moralischen Gesinnung entsprechende Glück. Die Einheit von Moralität und Glück ist somit der Gegenstand der reinen praktischen Vernunft. Kant faßt „die unbedingte Totalität des Gegenstandes der reinen praktischen Vernunft, unter dem Namen des höchsten Guts. “2 Diesen Begriff nennt er unmittelbar anschließend eine „Idee. ${ }^{\text {*3 }}$ Ideen sind für Kant wie in der ersten, so auch in der zweiten Kritik dialektisch und bedürfen der Kritik. So wird einzig diese Idee in zweiten Buch des ersten Teils der Kritik der praktischen Vernunft erörtert. Diese Erörterung wird notwendig, weil in der Idee des höchsten Gutes zwei Begriffe, und zwar als Grund und Folge notwendig verbunden gedacht werden, nämlich Tugend und Glückseligkeit. Eine Verbindung ist entweder analytisch oder synthetisch. Eine analytische Verbindung würde beide Begriffe so verbinden, daß der eine sich aus dem anderen ergäbe, also mit diesem identisch wäre; das aber ist hier nicht der Fall. Also muß die Verbindung synthetisch sein. Diese Synthesis wird als praktisch notwendig, also nicht empirisch nachweisbar gedacht. Moralität beruht auf Freiheit, sowohl als Autonomie der Vernunft, wie als Selbstbestimmung des Willens, Glück dagegen ist der Natur zu verdanken. Im höchsten Gut ist demnach die synthetische Einheit von Freiheit und Natur zu denken. Die Lösung des Problems stellen für Kant bekanntlich die Postulate Gottes und der Unsterblichkeit dar. Durch diese Postulate ist gedacht, daß die Natur entsprechend der praktischen Vernunft gestaltet ist und Glück gewähren kann. Damit wird zumindest dem Gedanken nicht widersprochen, es sei „doch immer nur eine und dieselbe Vernunft, die, sei es in theoretischer oder praktischer Absicht, nach Principien a priori urtheilt"“4 . Die Einheit der Vernunft ist also Problem, und zwar unter dem Primat der praktischen Vernunft.

\footnotetext{
${ }^{1}$ Immanuel Kant, Critik der practischen Vernunft (Riga: Hartknoch, 1788), 235 (ferner zitiert als $\mathrm{KpV}$ ); Immanuel Kant, Kritik der praktischen Vernunft, Schriften V, in Kant's Gesammelte Schriften „Akademieausgabe“, Königlich Preußische Akademie der Wissenschaften, Berlin 1900 ff. (bisher 29 Bände), Reimer, ab 1922 de Gruyter, 130 (ferner zitiert als AA V).

${ }^{2}$ Kant, KpV 194 (AA V, 108).

${ }^{3}$ Kant, KpV 194 (AA V, 108).

${ }^{4}$ Kant, KpV 218 (AA V, 121).
} 
An der vorhin zitierten Stelle, an der Kant auf das höchste Gut zu sprechen kommt, fährt er fort: „Diese Idee [nämlich des höchsten Guts] praktisch, d. i. für die Maxime unseres vernünftigen Verhaltens, hinreichend zu bestimmen, ist die Weisheitslehre. ${ }^{5}$ Diese Lehre sagt also, und zwar hinreichend, wie wir die Maxime unseres Willens als vernünftige Wesen bestimmen sollen; wenn Kant hier von der Maxime im Singular spricht, so darf man diese als die schlechterdings grundlegende Maxime begreifen. Das entspricht der Idee, die ja auch nicht Handlungsanweisungen meint, sondern solche als auf das höchste Gut gerichtet ermöglicht. Weisheitslehre unterrichtet demnach nicht nur darin, was die Pflicht gebietet, sondern auch darin, wie denn die Natur entsprechend dem sittlichen Willen so gestaltet werden kann, daß sie Glück gewährt. Da der sittliche Wille das Allgemeine will, ist hier nicht nur an individuelles, sondern an allgemeines Glück zu denken.

Mit der hier genannten Weisheitslehre ist eine empirisch erworbene gemeint; denn diese ,als Wissenschaft ist Philosophie“"6, wie Kant sagt. Er erläutert, Philosophie sei „eine Anweisung zu dem Begriffe [...] worin das höchste Gut zu setzen, und zum Verhalten, durch welches es zu erwerben sei." ${ }^{67}$ Kant betont nochmals, Philosophie sei zu fassen, ,als eine Lehre vom höchsten Gut, so fern die Vernunft bestrebt ist, es darin zur Wissenschaft zu bringen. “8 Philosophie versteht Kant als kritisch reflektiertes, letztlich systematisches Wissen, hier also der Weisheitslehre.

Philosophie ist demnach wissenschaftliche Weisheitslehre, deren Gegenstand das höchste Gut oder anders die Einheit der Vernunft unter dem Primat der praktischen ist. Mit dieser Erinnerung an Kant, kann ich nun zu Schellings Reflexion übergehen.

\section{SCHELLINGS REDE VON WEISHEIT}

Von Weisheit redet Schelling nicht nur, aber jedenfalls ausführlich in der Philosophie der Offenbarung, so daß ich mich auf diese Texte in angemessener Zeit beziehen kann. Die Philosophie der Offenbarung wurde von Schellings Sohn Karl Friedrich August 1858 als die Bände III und IV der Nachgelassenen Schriften, auch gezählt als Bände XIII und XIV der Sämmtlichen Werke, herausgegeben. Es handelt sich um eine späte Fassung aus Schellings letzten Jahren. Zuerst vorgetragen hat er die Philosophie der Offenbarung 1831/32 in München. Eine Abschrift des Manuskripts hat Walter E. Ehrhardt entdeckt und 1992 unter dem Titel Urfassung der Philosophie der Offenbarung herausgegeben. Auf beide Texte beziehe ich mich, vornehmlich auf die Urfassung.

\footnotetext{
${ }^{5}$ Kant, KpV 194 (AA V, 108).

${ }^{6}$ Kant, KpV 194 (AA V, 108).

${ }^{7}$ Kant, KpV 194 (AA V, 108).

${ }^{8}$ Kant, KpV 194 (AA V, 108).
} 
Am Beginn der Urfassung erläutert Schelling, was er lesen will, nämlich Philosophie der Offenbarung, wobei er zunächst erläutert, was Philosophie ist. Wie das griechische $\varphi \imath \lambda \varepsilon v$ ausdrückt, handelt es sich um ein Streben, ein Wollen. Schelling sagt, sie verlange, „daß sie etwas Gewisses mit Bewußtsein

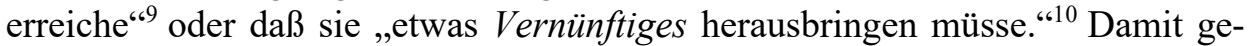
stehe man ihr ,einen vernünftigen Zweck zu. “" ${ }^{\text {11 }}$ Diese Erklärung reicht Schelling nicht, da vieles gar nicht aus Vernunft zu erklären sei. Daher bestimmt Schelling dasjenige „Wollen, welches die Philosophie leitet,“ als „ein sittliches Wollen [...], welches seinen Grund in der Freiheit hat." ${ }^{\text {"12 }}$ Und nun kommt es geradezu kantisch: „Philosophie heißt »Wollen der Weisheit«." Und zur Deutlichkeit fügt Schelling hinzu: „Nicht jede Erkenntnis, sondern nur die der Weisheit, genügt der Philosophie." ${ }^{13}$ Weisheit, so fährt er fort, unterscheide sich von Klugheit dadurch, daß diese auch Unsittliches wollen könne. Wer nur klug handle, ,sieht nie auf das wahre Ende, auf das eigentlich zuletzt oder in letzter Instanz seyn Sollende hinaus, sondern nur auf das jetzt und zunächst Mögliche. ${ }^{\text {"14 }}$ Dagegen richte sich Weisheit „auf das wahre, und eben deswegen bleibende Ende.“15 Ende ist hier, wie seinerzeit durchaus üblich, als Ziel verstanden. Wenn wir als das wahre Ziel Kants höchstes Gut, das für ihn letztes Ziel ist, einsetzen, bleibt nur noch nach der Naturbestimmung, dem Glück, zu fragen. Die Antwort gibt Schelling mit Kant: „Was [...] Gegenstand der Erkenntnis ist, muß das Gepräge des Erkennenden an sich haben, wie jedem einleuchtet, der die Kantische Theorie der Erkenntnis innehat. [...] Kant setzt einen an sich verstandlosen Stoff voraus, der aber das Gepräge des Verstandes hat." ${ }^{\text {"16 }}$ Sinnliche Daten werden durch Anschauungsformen und Kategorien geordnet. „Die erste Voraussetzung der Philosophie als Streben nach Weisheit ist also, daß in dem Gegenstand, d. h. daß in dem Seyn, in der Welt selbst Weisheit sey. “17 Schelling hebt das Wort Voraussetzung hervor; das will sagen, im Wollen der Weisheit sei schon antizipiert, daß dasjenige, was Gegenstand der Erkenntnis ist, dies nur soweit ist, ,als es selbst die Form und das Gepräge des Erkennenden schon an sich trägt,“ und Schelling fügt noch hinzu ,wie jedem einleuchten muß, der auch nur die Kantsche Theorie

\footnotetext{
${ }^{9}$ Friedrich W. J. Schelling, Urfassung der Philosophie der Offenbarung, hg. Walter E. Ehrhardt, 2 Bd. (Hamburg: Meiner, 1992), 19.

${ }^{10}$ Friedrich W. J. Schelling, Sämmtliche Werke, hg. K. F. A. Schelling, Zweite Abtheilung, Dritter Band (Stuttgart und Augsburg: J. G. Cotta'scher Verlag, 1858), im Folgenden SW XIII, hier SW XIII, 200.

${ }^{11}$ Schelling, Urfassung der Philosophie der Offenbarung, 20.

${ }^{12}$ Ibid., 21. Vgl. Schelling, Sämmtliche Werke, XIII, 201.

${ }^{13}$ Ibid., 21.

${ }^{14}$ Schelling, Sämmtliche Werke, XIII, 202.

${ }^{15}$ Schelling, Urfassung der Philosophie der Offenbarung 22. Vgl. Schelling, Sämmtliche Werke, XIII, 202.

${ }^{16}$ Schelling, Urfassung der Philosophie der Offenbarung, 23.

${ }^{17}$ Schelling, Sämmtliche Werke, XIII, 203.
} 
der Erkenntniß etwas geistreicher als gewöhnlich aufzufassen versteht. “" ${ }^{18}$ Diese Hinzufügung macht klar, daß Schelling sich nicht, wie bisweilen behauptet, von Kants Transzendentalphilosophie entfernt. Ohne Kants Bestimmung der Weisheit eigens anzuführen, stellt Schelling sie in seinen Worten dar.

Dann aber reflektiert Schelling Kants Gedanken. Erinnern wir uns, daß das höchste Gut den Gedanken eines Gottes, der Freiheit und Natur in Übereinstimmung bringt, postuliert. Schelling formuliert diesen Gedanken so: „Die Philosophie setzt ein Sein voraus, welches gleich anfangs mit Voraussicht, mit Freiheit, entsteht. ${ }^{\text {“19 }}$ Mit anderen Worten: „Die erste Voraussetzung der Philosophie ist, daß in dem Sein - in der Welt - Weisheit sei. ${ }^{\text {'20 }}$ Schelling erläutert: „Ich verlange Weisheit - heißt soviel - als ich verlange ein absichtlich gesetztes Sein.“21 Schelling wiederholt hier das Wort „verlangen“. Wer Weisheit will, würde seinem Wollen widersprechen, wenn er sie aus Notwendigkeit wollte. Im Wollen der Weisheit liegt also schon das Wollen der Freiheit, aus der das Sein gesetzt ist. Wollen ist hier selbstredend nicht als vernunftloser Trieb oder ähnlich gemeint, sondern als freies, vernünftiges Wollen. Gewollt wird hier das Wissen des wahren Endes.

Schelling überlegt weiter. Wer Weisheit, genauer reflektierte Weisheit, will, will das wahre Ende wissen. Ein Ende ist aber nicht zu begreifen ohne einen Anfang, ein Ziel nicht ohne einen Ursprung. Dem Sein, welchem ein Ziel zugedacht wird, muß auch ein Ursprung, und da Zielsetzung nur für Vernunft und Freiheit gedacht werden kann, dem Ziel Freiheit vorausgedacht werden. Dann aber ist das Sein nicht aus sich selbst zu begreifen. Gegenstand der Philosophie ,ist also nicht das Sein selbst, sondern das, was vor dem Sein ist, um eben das Sein zu begreifen. Hiermit“, fährt Schelling fort, „habe ich Sie [die Hörer] in den Anfang der Philosophie gestellt.“222

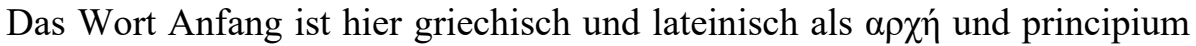
zu hören. Anfang ist Ursprung, Prinzip, Grund und auch Herrschaft. Der Anfang ist dem, was angefangen hat, voraus, nicht unbedingt zeitlich, aber doch als Prius gedacht. Nun richtet sich aber unser Erkennen auf das, was ist; dieses ist der Gegenstand unseres Wissens. Hier aber soll das, was vor dem Sein ist, gedacht werden. Schelling hält dies offensichtlich für schwierig; denn er fordert seine Hörer explizit auf, Ihre „Aufmerksamkeit ganz auf diesen Begriff »dessen, was vor dem Seyn ist, « zuzuwenden." ${ }^{\text {23 }}$ Da Schelling uns zumutet, Kant etwas geistreicher zu verstehen als gewöhnlich, sehen wir, daß er von den Problemen

\footnotetext{
${ }^{18}$ Ibid.

${ }^{19}$ Schelling, Urfassung der Philosophie der Offenbarung, 23.

${ }^{20}$ Ibid.

${ }^{21}$ Ibid.

${ }^{22}$ Ibid.

${ }^{23}$ Schelling, Sämmtliche Werke, XIII, 204. Vgl. Schelling, Urfassung der Philosophie der Offenbarung, 23.
} 
der transzendentalen Dialektik redet. Dies wird deutlich, wenn er die Zuwendung problematisiert. Das, was vor dem Sein ist, ist ,nämlich nichts im Vergleich mit dem, was hernach seyn wird, oder im Verhältniß zu dem Seyn, über das wir eben hinausgegangen sind, das wirkliche. ${ }^{\text {“24 }}$ Wir erinnern uns, daß in Kants transzendentaler Dialektik die Ideen nichts Erkennbares, gewissermaßen nichts im Vergleich mit den wirklichen Gegenständen sind. Allerdings redet Kant von den Ideen, insofern sie in Bezug auf die Erkenntnisse gedacht werden müssen. Am deutlichsten wird dies bei der Gottesidee, die eine Schematisierung der omnitudo realitatis, der Allheit der Sachbegriffe, ist. Diese omnitudo muß nämlich gedacht werden, wenn wir Prädikation verstehen wollen, weil die Zuschreibung eines Prädikats eben dieses aus der Totalität aller möglichen Prädikate herausgreift. Die Idee Gottes ermöglicht keine Gotteserkenntnis, sondern ist die Bedingung der Möglichkeit der Prädikation in unserem Erkennen; sie ist in Schellings Worten „vor“ dem Prädizierten und wird als Bedingung der Möglichkeit gerade für und damit vom Prädizieren her gedacht. ${ }^{25}$

Schelling faßt diesen Gedanken so: „Obgleich [...] hinausgegangen über das Seyn, betrachten wir das, was vor dem Seyn ist, doch nur in Bezug auf eben dieses Seyn, denn ein anderes Mittel es zu bestimmen [...] gibt es für uns nicht. “' ${ }^{\prime 2}$ Kantisch gewendet heißt dies: Wir denken die Bedingung der Möglichkeit, um das Bedingte zu begreifen. Bei Schelling liest sich das so: Weil wir das, was vor dem Sein ist, „denken oder setzen, eigentlich nicht zunächst um es selbst und an sich kennen zu lernen, sondern um das Seyn aus ihm zu begreifen, so ist es für uns auch vollkommen hinreichend, es vorerst nur in Bezug auf dieses Seyn zu bestimmen. “27 Das ist durchaus kantisch reflektiert; Ideen und Postulate werden gedacht um faktisches Erkennen und Handeln begreifen zu können.

Schellings nächster Schritt verwundert zunächst einmal. Er läßt sich so einführen. Wenn das, was vor dem Sein ist, als dessen Ursprung verstanden werden muß, dann ist das Sein das Folgende. So gedacht ist also ein Ursprung von etwas, das noch gar nicht ist, gedacht. Wenn also, wie gerade zitiert, das, was vor dem Sein ist, nur in Bezug auf dieses Sein bestimmt werden kann, dann ist das Sein gedacht als das, was noch gar nicht ist. Es ,ist das noch nicht Seyende, aber das seyn wird. “28 Die Urfassung formuliert etwas deutlicher: „Wir wollen das Sein begreifen - also müssen wir das, was vor dem Sein ist, in bezug

\footnotetext{
${ }^{24}$ Schelling, Sämmtliche Werke, XIII, 204. Vgl. Schelling, Urfassung der Philosophie der Offenbarung, $23 \mathrm{f}$.

${ }^{25}$ Vgl. hierzu Wolfgang Hogrebe, Prädikation und Genesis. Metaphysik als Fundamentalheuristik im Ausgang von Schellings 'Die Weltalter' (Frankfurt a. M.: Suhrkamp, 1989).

${ }^{26}$ Schelling, Sämmtliche Werke, XIII, 204. Vgl. Schelling, Urfassung der Philosophie der Offenbarung, 24.

${ }^{27}$ Ibid. Vgl. Schelling, Urfassung der Philosophie der Offenbarung, 24.

${ }^{28}$ Ibid., 204.
} 
auf das künftige Sein begreifen. ${ }^{\text {} 29}$ Wenn man Kant brav studiert hat, fühlt man sich jetzt aufs Glatteis gelockt, zumal es dann auch noch heißt: „Der Ausgangspunkt der Philosophie ist also das, was sein wird, das absolut Zukünftige: es ist also unsere Aufgabe, in die Wesenheit des absolut Zukünftigen einzudringen. “30 Der Ausweg, Zukunft hier als metaphorische Rede zu verstehen, hilft nicht; die Rede ist ernst gemeint. Zunächst können wir braven Kantianer uns damit helfen, daß wir in der Kritik der reinen Vernunft lesen, „daß der Begriff der Veränderung und mit ihm der der Bewegung [...] nur durch und in der Zeitvorstellung möglich ist; daß, wenn diese Vorstellung nicht Anschauung (innere) a priori wäre, kein Begriff, welcher es auch sei, die Möglichkeit einer Veränderung, d. i. einer Verbindung contradictorisch entgegengesetzter Prädicate [...] in einem und demselben Objecte, begreiflich machen könnte. Nur in der Zeit können beide contradictorisch-entgegengesetzte Bestimmungen in einem Dinge, nämlich nach einander, anzutreffen sein." ${ }^{31}$ Brav, wie wir sind, wissen wir auch, daß Kant hier von anschaulichen Gegenständen, die nur in der Zeit sein können, spricht, nicht aber von etwas nur Gedachtem.

Nun läßt sich immerhin verstehen, daß Schelling hier von einem realen Werden, nämlich des Seins aus dem, was vor dem Sein ist, sprechen will. Dieses Werden ist zweifellos nicht anschaulich, aber doch als ein reales Werden zu denken. Ob ein solches nun anschaulich oder nicht ist, so müssen einem und demselben Ding oder Begriff contradiktorische Prädikate zugeschrieben werden. Es scheint also notwendig, die Zeit nicht nur für Anschauliches, sondern auch für Gedachtes in Ansatz zu bringen. Bei der Willensbestimmung sind jedenfalls einem und demselben Subjekt kontradiktorische Bestimmungen zuzuerkennen, zuerst nicht bestimmt sein und dann bestimmt sein. Nun wissen wir wohl, daß Kant die Willensbestimmung außerhalb der Zeit setzt, weil er sie sonst nur als unfreie Naturkausalität verstehen kann, aber nicht nur wir wissen das, auch Schelling weiß das und stimmt Kant darin zu. Andererseits muß, wenn eine reale geistige Bewegung gedacht werden soll, die Möglichkeit von einer kontradiktorischen Bestimmung zu einer anderen überzugehen, mitgedacht werden. Folglich denkt Schelling eine die Bewegung implizierende Zeit, welche allerdings nicht die der Anschauung ist.

Was veranlaßt ihn dazu? Vermutlich ist es der Begriff von Weisheit und der damit zusammenhängende des höchsten Guts, den er bei Kant vorfindet. Dieses ist zu denken möglich unter der Bedingung des Gottespostulats. Gott wird so gedacht, daß er die Möglichkeit der Harmonie von sittlicher Freiheit und Glück ermöglicht. Der hier gedachte Freiheitsbegriff ist nicht der transzendentale,

\footnotetext{
${ }^{29}$ Schelling, Urfassung der Philosophie der Offenbarung, 24.

${ }^{30}$ Schelling, Urfassung der Philosophie der Offenbarung, 24.

${ }^{31}$ Immanuel Kant, Kritik der reinen Vernunft, in Kant's Gesammelte Schriften „Akademieausgabe“, Königlich Preußische Akademie der Wissenschaften, Berlin 1900 ff. (bisher 29 Bände), Reimer, ab 1922 de Gruyter, KrV B 48 f. (AA III, 59).
} 
sondern der praktische. Praktische Freiheit ist Wollen. Dann ist hier zu denken, daß Gott eine Welt will, die eine Harmonie von Freiheit und Natur zuläßt. Die Kantische Vorgabe ist Schelling präsent. Die Frage ist nur, wie der Zusammenhang von Freiheit und Natur, den Gott garantiert, genau zu denken ist. Schelling bezweifelt nicht die von Kant gedachte Garantie des Zusammenhangs von Freiheit und Natur durch Gott; er fragt, wie diese genau zu denken sei.

Um diese Frage zu beantworten, entwickelt er seine Potenzenlehre, deren Darstellung und Erläuterung eine weit über den hier zu Verfügung stehenden Raum beanspruchte. Ich erläutere in aller Kürze von Kant her so: Im Gottespostulat muß Gott als freier Herr der Natur gedacht werden, da er Glück zuteilen kann. Als Herr muß er - in Schellingscher Sprache - ,vor dem Sein' gedacht werden. Wird er als Herr gedacht, so ist Freiheit das Erste und die Notwendigkeit der Natur das Zweite. Seine Potenzenlehre entwickelt Schelling nun gerade dazu, Gott als frei und damit aus Freiheit denken zu können.

Die daraus resultierende Aufgabe besteht darin, ein Modell zu entwickeln, das die Schöpfung, Natur und endliche Freiheit, als freie Tat denken läßt. Eine freie Tat ist eine bewußte Tat, eine deren Telos gedacht ist, bevor es realisiert ist. Diesen Gedanken der Schöpfung als Telos denkt Schelling als Bedingung der Möglichkeit von freier Schöpfung und nennt ihn Weisheit. Wenn nun Philosophie Streben nach Weisheit ist, so genau nach jener, die das wahre Ende kennt; denn das höchste der Geschöpfe, der Mensch, sollte sich der freien Tat, der er sich verdankt, nicht nur als Anfang, sondern als ganzer Prozeß bewußt sein. Wenn dieser Prozeß einer der Freiheit sein soll, muß der Mensch Mitwisser $^{32}$ dieses Prozesses sein, da er diesen Prozeß mitgestaltet.

Einen solche Mitwisserschaft dokumentiert sich für Schelling im Buch der Weisheit $^{33}$, das teils zum Alten Testament gerechnet wird, teils als apokryph gilt, weil es nicht in hebräischer, sondern in griechischer Sprache verfaßt wurde. Es ist höchstwahrscheinlich im ersten Jahrhundert vor Christus in Alexandrien entstanden. Man kann es daher als entstanden aus griechischer Philosophie und Offenbarung ansehen, und es stellt sich die Aufgabe, die Offenbarung zu begreifen, den Prozeß der Freiheit nicht hinzunehmen, sondern zu begreifen und so mitzuvollziehen.

Weisheit ist somit für Schelling Mitwisserschaft des Anfangs und des Endes des Prozesses der Freiheit, Philosophie ist das Streben nach Weisheit, nämlich diese ursprüngliche Mitwisserschaft so gründlich als möglich zu begreifen. ${ }^{34}$

Schelling reflektiert, wie gezeigt werden sollte, Weisheit, wie er es bei Kant schon gefunden hatte, als Wissen des wahren Endes, bzw. Zieles.

\footnotetext{
${ }^{32}$ Vgl. Schelling, Urfassung der Philosophie der Offenbarung,126.

${ }^{33}$ Vgl. Ibid., 132-134.

${ }^{34}$ Vgl. Ibid., 127.
} 


\section{BIBLIOGRAPHIE}

Hogrebe, Wolfgang. Prädikation und Genesis. Metaphysik als Fundamentalheuristik im Ausgang von Schellings 'Die Weltalter'. Frankfurt a. M: Suhrkamp, 1989.

Kant, Immanuel. Critik der practischen Vernunft. Riga: Hartknoch, 1788.

- Kritik der praktischen Vernunft. Kant's Gesammelte Schriften „Akademieausgabe“, Königlich Preußische Akademie der Wissenschaften, Berlin 1900 ff. (bisher 29 Bände), Reimer, ab 1922 de Gruyter.

- Kritik der reinen Vernunft. Kant's Gesammelte Schriften „Akademieausgabe“, Königlich Preußische Akademie der Wissenschaften, Berlin 1900 ff. (bisher 29 Bände), Reimer, ab 1922 de Gruyter.

Schelling, Friedrich Wilhelm Joseph. Sämmtliche Werke, hg. K. F. A. Schelling, Zweite Abtheilung, Dritter Band. Stuttgart und Augsburg: J. G. Cotta'scher Verlag, 1858.

- Urfassung der Philosophie der Offenbarung, hg. Walter E. Ehrhardt, 2 Bd. Hamburg: Meiner, 1992.

\section{SCHELLING'S REFLECTION ON KANT'S NOTION OF WISDOM}

Kant characterizes wisdom as knowledge of the will of the highest good and appropriateness of the will to this good. He understands the highest good as a union of morality and happiness. Since these are not to be conceived upon the same lawfulness (Gesetzlichkeit), Kant thinks of the mediation of the postulates of immortality and of the existence of God. To determine, in a sufficient form, the idea of the highest good practically, i.e., as the maxim of our rational behavior, is for Kant the wisdom doctrine; in scientific reflection it is philosophy. Schelling agrees with that; for him, too, philosophy is the will of wisdom. Wisdom looks at the ultimate goal, the one which should actually come about. To know this and to make it the determining reason of the will is wisdom for Schelling.

\section{Keywords:}

Schelling, Kant, wisdom 